\title{
In vivo total body water assessment by total body electrical conductivity in rats suffering perturbations of water compartment equilibrium
}

\author{
BY N. BATTISTINI ${ }^{1}$, F. VIRGILI ${ }^{2}$, G. BEDOGNI', G. R. GAMBELLA \\ AND A. BIN I' \\ ${ }^{1}$ Institute of Human Physiology and Pathology, University of Modena, via Campi 287, 41100 \\ Modena, Italy \\ ${ }^{2}$ National Institute of Nutrition, via Ardeatina 546, 00178 Rome, Italy \\ ${ }^{3}$ Institute of Pathology, University of Genova, via L. B. Alberti 2, 16132 Italy
}

(Received 18 June 1992 - Accepted 27 October 1992)

\begin{abstract}
Total body electrical conductivity (TOBEC) is a simple and non-invasive method for the assessment of body composition in vivo. Information regarding the applicability of TOBEC in the condition of abnormal fluid balance is scarce. In the present paper we give the results of the comparison between TOBEC and total body water (TBW; assessed by the tritium dilution technique) in three groups of animals: (1) healthy $(n$ 17), (2) expanded fluid volume by secondary biliary cirrhosis (SBC; $n$ 9) and (3) Furosemide ${ }^{\circledR}$-treated rats $(n 9)$. The TOBEC score and TBW by tritium dilution were found to be highly correlated in the pooled sample $(r 0.90)$ and in normal $(r 0.87)$, SBC $(r 0.73)$ and Furosemide-treated $(r$ 0.89) rats. However, the relationship between TOBEC and TBW, described by least-squares regression analysis, was found to be similar for $\mathrm{SBC}$ and normal rats but was significantly different for Furosemide-treated and normal rats. These findings suggest that TOBEC is unable to track TBW accurately when the ratio between intracellular and extracellular water is chronically or acutely altered.
\end{abstract}

Total body water: Tritium dilution space: Total body electrical conductivity.

Total body electrical conductivity (TOBEC) is a simple and non-invasive method proposed for the assessment of body composition in vivo (Lukaski, 1987). TOBEC technique relies on the difference in electrical conductivity and dielectric properties between fat-free mass (FFM; which actually contains all the water and conducting electrolytes) and fat mass, and has been demonstrated to predict accurately the FFM and the total body water (TBW) in different species of animals and in humans (Domermuth et al. 1976; Bracco et al. 1983; Klish et al. 1984; Van Loan \& Maycin, 1987). The electrical conduction of a living organism exposed to an oscillating radio frequency is related to the water and electrolyte distribution in the biological conductor. Each electrolyte contributes differently to the conductivity, $\mathrm{H}_{2} \mathrm{PO}_{4}^{-}$being less conductive than $\mathrm{Cl}^{-}$in test bottles filled with ion solutions placed in a TOBEC instrument (Cunningham et al. 1986). This difference results in a specific score for intra- (ICW) and extracellular water (ECW) compartments. Thus, acute or chronic perturbations of body fluid compartments (such as generalized oedema or dehydration) could theoretically affect the TOBEC measurements. Only a few studies have been described in which TOBEC was related to TBW in the condition of abnormal fluid balance. TOBEC has been reported to predict consistently TBW following drug-induced diuresis, severe burns and low-protein diets in rats (Cunningham et al. 1986) and after intraperitoneal injections of saline $(9 \mathrm{~g} \mathrm{NaCl} / 1)$ in miniature pigs (Cochran et al. 1989).

In the present study we have compared TOBEC and tritium dilution space measurements 
in healthy rats and in situations of chronic or acute abnormal body fluid balance, i.e. dehydration induced by Furosemide treatment and interstitial fluid accumulation due to secondary biliary cirrhosis (SBC).

\section{MATERIALS AND METHODS}

Surgical treatment. Nine weanling male Sprague-Dawley rats underwent bile duct ligation as described elsewhere (Accattino et al. 1981). At 4 weeks after surgery the mean weight of rats was 461 (SD 54) $\mathrm{g}$ (range 375-560 g) and the TOBEC and tritium dilution protocols were performed as described previously. Liver histological examination, performed at the death of the animals (after TOBEC and tritium analysis), confirmed the biliary cirrhosis and an evident water retention.

Furosemide treatment. Nine healthy male Sprague-Dawley rats (mean weight 426 (SD 22) g; range 397-461 g) were dehydrated by giving an intraperitoneal injection of $7 \mathrm{mg}$ Furosemide (Lasix ${ }^{\circledR}$; Hoechst Ag. Frankfurt, Germany)/kg. The administration of this drug resulted in an average diuresis of 17.0 (SD 1.5) $\mathrm{g}$ in $2 \mathrm{~h}$. At the end of this $2 \mathrm{~h}$ period TOBEC and tritium dilution protocols were performed as described later (below).

Control rats. Seventeen healthy male Sprague-Dawley rats (mean weight 402 (SD 75) g; range $261-478 \mathrm{~g}$ ) were used as control animals and underwent the same protocol except the bile duct ligation or Furosemide administration

TOBEC and tritium dilution protocol. Tritiated water $(5 \mu \mathrm{Ci}$; Amersham International Plc, Amersham, Bucks.) was given intraperitoneally and $30 \mathrm{~min}$ after tritium administration, rats were weighed, anaesthetized with diethyl ether and underwent TOBEC measurement (EM-Scan SA2, Springfield, IL, USA) at $5 \mathrm{MHz}$ oscillating current. TOBEC value was taken as the mean of three or more different measurements as suggested by the manufacturer. The coefficient of variation of different TOBEC readings was $<5 \%$. No attempts were made to derive TBW or lean mass from previously published formulas and, therefore, TOBEC reading was considered per se as the instrument score.

A sample of blood was taken by cardiac puncture, while the rats were still anaesthetized, 30-40 min after tritium administration. Blood was immediately centrifuged and portions of plasma and administered dose were counted for $\beta$ activity; TBW was calculated from the dilution of the amount of tritium injected divided by 1.03 (Sheng \& Huggins, 1979, 1989) and corrected for the amount of water in plasma (Bauer et al. 1975).

Statistical analyses (means and standard deviations, least-squares regression analysis and ANOVA) were performed using a Statview ${ }^{\circledR}$ package for Macintosh ${ }^{\circledR}$ P.C.

\section{RESULTS}

The results of TOBEC measurements are given in Table 1. The control group included animals with a wider range of weight (402 (SD 75) g; range 261-478 g) than SBC (461 (SD 54) g; range 375-560 g) and furosemide-treated animals (426 (SD 22) g; range $397-461 \mathrm{~g})$. Body hydration was significantly $(P<0.0001)$ higher in SBC animals than in control rats, confirming that secondary biliary cirrhosis induced a significant fluid accumulation.

The absolute TOBEC score, and the TOBEC score expressed on a per g body weight basis, were also significantly higher in SBC and Furosemide-treated animals than those of the control group (see Table 1).

Fig. 1 shows TOBEC v. TBW for SBC animals, Furosemide-treated and healthy rats. The correlation coefficient for the pooled sample (SBC plus Furosemide-treated plus controls) was rather high $(r 0 \cdot 90)$ indicating that TOBEC was positively associated with the water content of the animals. 


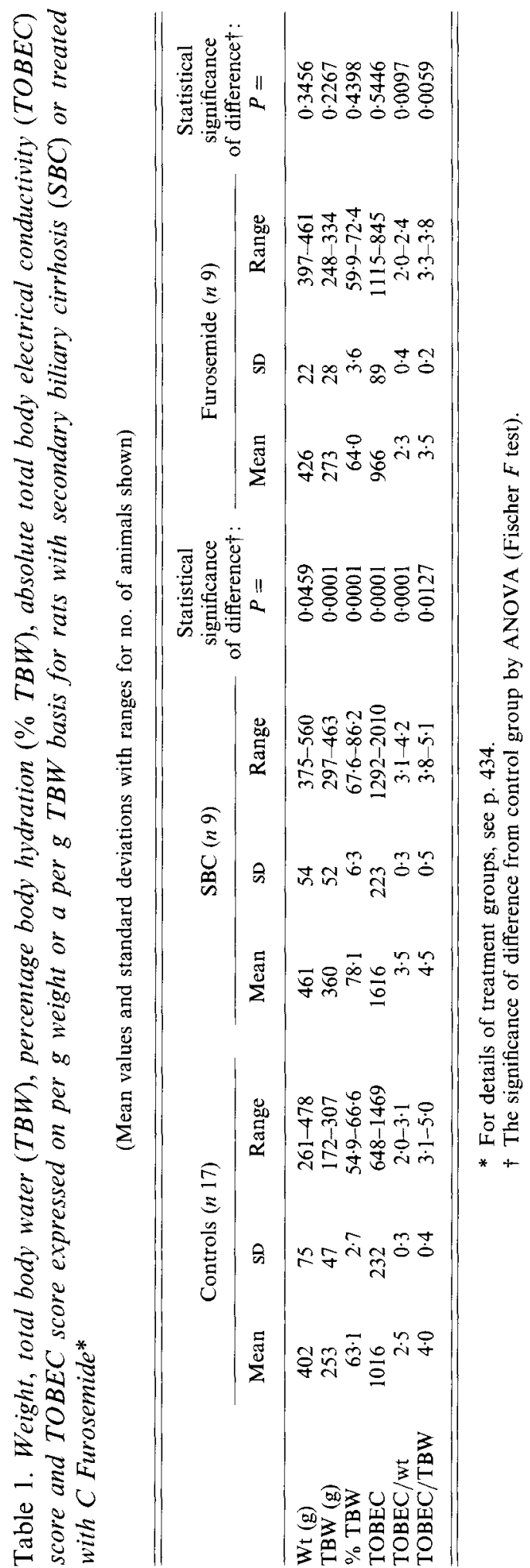




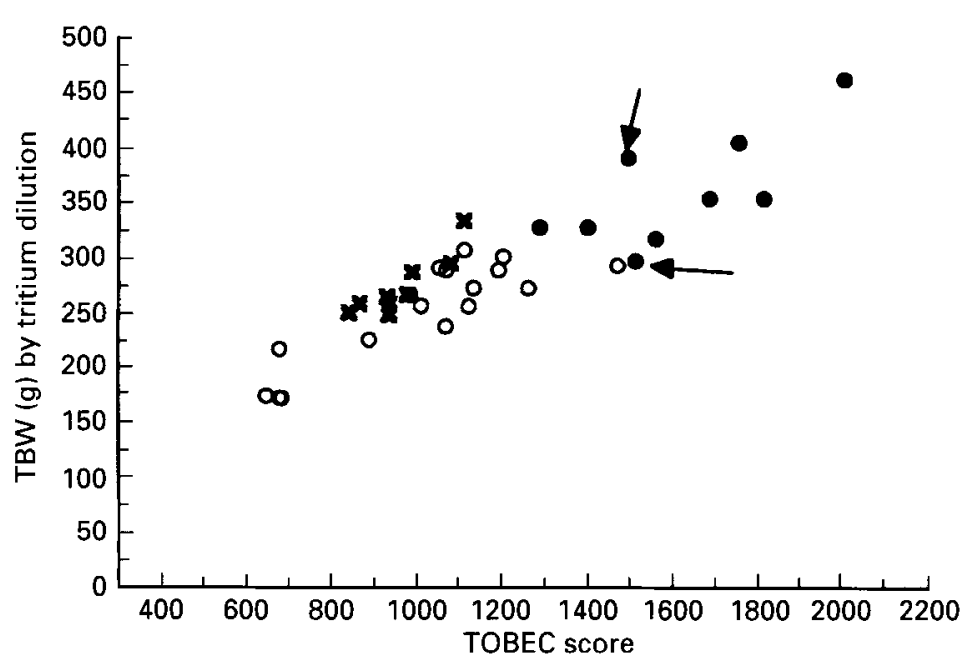

Fig. 1. Correlation between total body electrical conductivity (TOBEC) score and total body water (TBW) in (O) rats with secondary biliary cirrhosis $(\mathrm{SBC}) ;(X)$, Furosemide-treated and $(O)$ control rats. For details of treatment groups, see p. 434. Correlation coefficients: controls $0.87, \mathrm{SBC} 0.73$, Furosemide-treated 0.89 , all rats 0.90 . The two arrows indicate two SBC rats with an equal TOBEC score and different TBW.

The analysis of the least-squares linear regression of TOBEC scores $v$. TBW, for SBC, Furosemide-treated and control groups gives the following formulas:

healthy rats: TBW $(\mathrm{g})=0 \cdot 17 \times$ TOBEC $+79 \cdot 2 ; r 0.87 ; P<0.0001$; SE of the prediction (SEE) $2.6 \mathrm{~g}$

SBC: TBW $(\mathrm{g})=0.17 \times$ TOBEC $+84 \cdot 3 ; r 0.73 ; P<0.02 ;$ SEE $5.0 \mathrm{~g}$

Furosemide: TBW $(\mathrm{g})=0.28 \times$ TOBEC $+6.5 ; r 0.89 ; P<0.001 ;$ SEE $2.0 \mathrm{~g}$.

SBC and control rats have identical values for the slope and slightly different intercepts, the regression line for TOBEC $v$. TBW in SBC being parallel to the line obtained for the controls but displaced by $5 \mathrm{~g}$. On the other hand, the relationship between TOBEC score of furosemide-treated animals and tritium dilution space is described by rather different variables.

The similarity between the variables for the regression lines obtained for SBC and control rats indicates that the decrease in $\mathrm{ICW}: \mathrm{ECW}$ resulting from the accumulation of interstitial water is reflected by a proportional increase in electrical conductivity. The difference between intercepts is confirmed by the observation that TOBEC:TBW was significantly higher for SBC than for control animals (4.5 (SD 0.5) and 4.0 (SD 0.4) respectively; $P<0.0127$; see Table 1). This can be explained by assuming that the electrolyte compositions of the intracellular and extracellular compartments are quite distinctive. Water and electrolyte balance is seriously compromised in cirrhosis and the increase of interstitial fluid is accompanied by a reduction in the concentration of cation and protein anions with respect to ICW and plasma (Klish et al. 1984).

After the acute diuresis induced by Furosemide, the relationship between TOBEC and TBW appears completely different. In these animals the least-square linear regression analysis generated a slope $(0 \cdot 28)$ significantly steeper than in both controls and SBC animals $(0 \cdot 17)$, indicating that a unit of TOBEC corresponds to an increase in TBW almost twofold greater in SBC than in control rats. This is confirmed by the significant difference $(P<0 \cdot 0059)$ between the TOBEC:TBW values for normal rats and Furosemide-treated rats $(4.0(\mathrm{SD} 0 \cdot 4)$ and $3.5(\mathrm{SD} 0 \cdot 2)$ respectively; see Table 1$)$. 


\section{DISCUSSION}

Our observations are in partial contrast with the findings of Cunningham et al. (1986) who reported TOBEC to reflect accurately the bidirectional changes induced by burns and Furosemide administration. Although the parameters of least square regression for treated animals and controls were not presented separately, Cunningham et al. (1986) concluded that TOBEC accurately predicts ECW, but not ICW when the ratio between the compartments falls below normal such as in the case of burns or malnutrition. Cunningham et al. (1986) reported that, following $50 \%$ body surface area burn, ICW:ECW is significantly decreased with respect to the controls. This fall would be caused by an increase in ECW and a parallel loss of ICW, expressed respectively as $\mathrm{Na}$ and $\mathrm{K}$ space.

The observations of Cunningham et al. (1986) have more recently been corroborated by Cochran et al. (1989), who reported that the expansion of extracellular fluid volume induced by intraperitoneal injections of physiological saline did not significantly affect both TBW and FFM prediction from TOBEC measurements.

These findings suggest that TOBEC score is probably mainly associated with the contribution of extracellular ions, while, as indicated by Cunningham et al. (1986), total body $\mathrm{K}$ presents a much lower correlation with TOBEC. In this case, a fluid perturbation which simply increases the extracellular volume without any electrolyte unbalance is unlikely to affect the prediction of FFM and TBW from TOBEC measurements (Cochran et al. 1989).

SBC animals could be suitable models to challenge the TOBEC method in situations of chronically-altered (decreased ICW:ECW) fluid and electrolyte balance. Findings reported here show that the slope of the least square regression analysis of TBW $v$. TOBEC score in $\mathrm{SBC}$ rats is identical to that obtained in controls. On the other hand, the intercept of the regression formula and TOBEC: TBW in SBC rats is slightly but significantly higher than that of control animals (see Table 1). The prediction of TBW from TOBEC score on the basis of a formula obtained on normally-hydrated animals would consistently be slightly but significantly biased.

The administration of the diuretic Furosemide disrupts the balance of body fluid compartments in the opposite direction (i.e. to an increase of ICW:ECW due to micturition, by inhibiting $\mathrm{Na}^{+}$and $\mathrm{Cl}^{-}$reabsorption in the medullary thick ascending limb of the loop of Henle, and induces a transient electrolye imbalance due to the inhibition of the $\mathrm{Na}^{+}-\mathrm{K}^{+}$exchange. In this case the correlation between TOBEC and TBW remains very high but the parameters that describe the relationship change significantly with respect to the control group, each unit of TOBEC having a greater TBW equivalence than in the control group.

Concerning the use of TOBEC to predict TBW, it is noteworthy that, in spite of a satisfying level of association, the wide scatter of points, both in SBC and control rats, suggests that TBW predicted from TOBEC would be quite imprecise. The mean of residuals is about zero, but the standard deviation of the mean is fairly high $(27 \mathrm{~g})$. This means that individuals with a similar TOBEC reading can have a TBW that is substantially different. This is particularly evident in SBC rats (see arrows in Fig. 1) where, for example, the two animals present an equal TOBEC score (1498 and 1510) and different TBW (391 and $297 \mathrm{~g}$ respectively). In SBC rats, the residuals of the regression present a weak correlation $(r 0.63)$ with the percentage body hydration, suggesting that the burden of water accumulation due to the induced cirrhosis is responsible for the scatter of SBC data-points presented in Fig. 1.

Even though in the present study we were not able to measure ECW or the extracellular ion concentration directly and compare them with TOBEC score, our results confirm that 
TOBEC is a valid and potentially useful method for the measurement of TBW in vivo. However, conditions that alter the ICW:ECW compartments chronically or acutely must be considered carefully to avoid potential errors.

This study was supported by a grant of the Ministero dell' Università e della Ricerca Scientifica e Tecnologica, special project 405: Free radical and redox state in pathology. The authors are indebted to Dr K. O'Sullivan for suggestions and revision of the manuscript.

\section{REFERENCES}

Accattino, L., Contreras, A., Berdicewski, E. \& Quintana, C. (1981). The effect of complete biliary obstruction on bile secretion, studies on the mechanism of postcholestatic choleresis in the rat. Journal of Laboratory and Clinical Medicine 97, 525-531.

Bauer, J. H., Willis, L. R., Burt, R. W. \& Grim, C. E. (1975). Simultaneous determination of plasma volume, red cell mass, extra-cellular fluid and total body water before and after volume expansion in dog and man. Journal of Laboratory and Clinical Medicine 86, 1009-1017

Bracco, E. F., Yang, M. U., Segal, K., Hashim, S. A. \& Van Itallie, T. B. (1983). A new method for estimation of body composition in live rat. Proceedings of the Society for Experimental Biology and Medicine 174, 143-146.

Cochran, W. J., Fiorotto, M. L., Sheng, H. P. \& Klish, W. J. (1989). Reliability of FFM estimates derived from total body electrical conductivity measurements as influenced by changes in extracellular fluid volume. American Journal of Clinical Nutrition 49, 29-32.

Cunningham, J. J., Molnar, J. A., Meara, P. A. \& Bode, H. H. (1986). In vivo total body electrical conductivity following perturbations of body fluid compartments in rats. Metabolism 35, 572-575.

Domermuth, W., Veum, T. L., Alexander, M. A., Hedrich, H. B., Clark, J. \& Eklund, D. (1976). Prediction of lean body composition of live market swine by indirect methods. Journal of Animal Science 43, 966-976.

Klish, W. J., Forbes, G. B., Gordon, A. \& Cochran, W. J. (1984). New method for the estimation of lean body mass in infants: validation in non-human models. Journal of Pediatric Gastroenterology and Nutrition 3, 199-204.

Lukaski, H. C. (1987). Methods for the assessment of body composition: traditional and new. American Journal of Clinical Nutrition 46, 537-556.

Sheng, H. P. \& Huggins, R. A. (1979). A review in body composition studies with emphasis on total body water and fat. American Journal of Clinical Nutrition 32, 630-647.

Sheng, H. P. \& Huggins, R. A. (1989). Tritiated water as a measure of total body water in immature rats growing at different rates. Journal of Applied Physiology 66, 476480.

Van Loan, M. \& Maycin, P. (1987). A new TOBEC instrument and procedure for the assessment of body composition: use of Fourier coefficients to predict lean body mass and total body water. American Journal of Clinical Nutrition 45, 131-137. 